\begin{tabular}{|} 
Ambiente \& Água - An Interdisciplinary Journal of Applied Science \\
ISSN 1980-993X - doi:10.4136/1980-993X \\
www.ambi-agua.net \\
E-mail: ambi.agua@gmail.com
\end{tabular}

\title{
Aplicação e análise comparativa de três protocolos de avaliação rápida para caracterização da paisagem fluvial
}

\author{
doi:10.4136/ambi-agua.1732
}

Received: 19 Aug. 2015; Accepted: 03 Nov. 2015

\author{
Jucimara Andreza Rigotti ${ }^{1 *}$; Cesar Augusto Pompêo ${ }^{2}$; \\ Alessandra Larissa D'Oliveira Fonseca ${ }^{3}$ \\ ${ }^{1}$ Universidade Federal do Rio Grande do Sul (UFRGS), Porto Alegre, RS, Brasil \\ Programa de Pós-Graduação em Recursos Hídricos e Saneamento Ambiental \\ ${ }^{2}$ Universidade Federal de Santa Catarina (UFSC), Florianópolis, SC, Brasil \\ Departamento de Engenharia Sanitária e Ambiental \\ ${ }^{3}$ Universidade Federal de Santa Catarina (UFSC), Florianópolis, SC, Brasil \\ Departamento de Geociências \\ *Autor correspondente: e-mail: anrigotti@gmail.com, \\ cesar.pompeo@ufsc.br, alarissa.fonseca@gmail.com
}

\section{RESUMO}

Os sistemas fluviais são dotados de características, como a heterogeneidade e a variabilidade, que permitem a qualificação de uma paisagem fluvial. Os protocolos de avaliação rápida vêm se mostrando ferramentas úteis para a caracterização da integridade, principalmente na escala de trecho de rio. O objetivo deste trabalho foi aplicar três protocolos e avaliar o desempenho deles entre si e em comparação com os resultados obtidos por métodos analíticos de avaliação da qualidade da água e da comunidade de macroinvertebrados aquáticos. O escalonamento multidimensional não-métrico (nMDS) foi aplicado com os parâmetros de qualidade da água e o Índice do Estado Trófico (IET), e com os dados de abundância de macroinvertebrados bentônicos. Também foram calculadas correlações entre índices e os resultados finais dos protocolos. A viabilidade de utilização dos protocolos para caracterização do habitat se confirmou, pois houve compatibilidade entre os resultados nos três procedimentos. Apesar das particularidades, todos abordam de alguma forma os aspectos gerais que caracterizam a integridade dos rios, indicando a condição do habitat que melhor suporta a biodiversidade aquática em rios urbanos.

Palavras-chave: integridade do habitat, qualidade da água, rios urbanos.

\section{Application and comparative analysis of three rapid assessment protocols to characterize fluvial landscape}

\section{ABSTRACT}

Fluvial systems are endowed with features such as heterogeneity and variability which allow the classification of a fluvial landscape. Rapid assessment protocols have been useful tools for the characterization of integrity, especially in river segment scale. The objective of this paper was to apply three protocols in order to evaluate their performance in comparison with each other and with the results obtained by analytical methods of water quality 
assessment and an evaluation of the aquatic macro-invertebrate community. Non-metric multidimensional scaling (nMDS) was applied using water quality parameters, trophic state index (IET), and abundance data of benthic macro-invertebrates. In addition, the study determined correlations between the index and the protocols' final scores. The protocols' feasibility for habitat characterization was confirmed, since the results were compatible for all procedures applied. Despite some particularities, each protocol offered a particular description of general aspects that affect river integrity, indicating which habitat conditions best support aquatic biodiversity in urban rivers.

Keywords: habitat integrity, water quality, urban streams.

\section{INTRODUÇÃO}

$\mathrm{Na}$ ecologia da paisagem o conceito de corredor, que pode ser aplicado aos cursos d'água, é considerado um dos padrões essenciais da organização da paisagem, através do qual se processam fluxos importantes de energia, de materiais e de espécies (Saraiva, 1999). O termo paisagem ribeirinha ou fluvial foi usado por Ward (1998) para indicar uma perspectiva global e de processos associados ao sistema fluvial. Os padrões de biodiversidade são direta e indiretamente influenciados pela geomorfologia das paisagens fluviais, as quais podem ser compreendidas como uma organização hierárquica aninhada (Ward, 1998). Tal hierarquia se inicia com a bacia hidrográfica e se particulariza pelas divisões em segmentos, trechos de rio e micro-habitat.

Sob a denominação de protocolos de bioavaliação rápida - Rapid Bioassessment Protocols - Barbour et al. (1999) agruparam uma série de métodos empregados pelas agências de recursos hídricos dos Estados Unidos. Segundo estes autores, os protocolos foram delineados inicialmente para ser ferramentas de baixo custo capazes de determinar a capacidade do rio para dar suporte à vida aquática. Essas ferramentas podem incluir a avaliação de aspectos físicos do habitat, regime de fluxo, qualidade da água e bioindicadores. Nos últimos anos um número bastante elevado de protocolos foi desenvolvido, em vários locais e com diferentes objetivos de avaliação. Para caracterizar o habitat, por exemplo, o trabalho de Fernández et al. (2011) analisaram cinquenta deles.

Em um sentido geral, os protocolos de avaliação rápida são formados por conjuntos de procedimentos, baseados em critérios estabelecidos previamente conforme a finalidade do estudo. Tal abordagem é constituída pela observação em campo, registrada na forma de descrição, sistema de pontuação ou de classificação. Os protocolos têm a possibilidade de ser rápidos em comparação com outros métodos de avaliação, como da fauna bentônica; contudo, não necessariamente utilizam apenas a avaliação visual. O procedimento em campo pode ser acompanhado de medições diretas, por exemplo, a estimativa de velocidade e profundidade presente no Índice do Habitat Fluvial - IHF (Pardo et al., 2002) ou incluir de antemão o uso de outras ferramentas, como mapas e imagens (Ollero et al., 2011). Além do mais, mesmo os protocolos idealizados para que sejam preenchidos completamente com observações em campo exigem um estudo detalhado, com desenvolvimento e adequação ao local de aplicação, como demonstraram Guimarães et al. (2012).

O resultado do uso dos protocolos pode fazer parte dos estudos ecológicos dos cursos d'água (Barbour et al., 1999) e de estratégias de monitoramento (Rodrigues e Castro, 2008), bem como estar presente no contexto de recuperação de rios (Habberfield et al., 2014), pois a aplicação dos mesmos é bastante efetiva no conhecimento do sistema fluvial de modo amplo. Nesse sentido, os protocolos se tornam instrumentos úteis, de baixo custo e relativa facilidade de aplicação, sendo tais aspectos também amplamente reconhecidos (Barbour et al., 1999; Callisto et al., 2001; Rodrigues e Castro, 2008). 
Este artigo trata da aplicação de três protocolos de avaliação rápida na escala de trecho de rio, com objetivo de avaliá-los quanto à caracterização da integridade da paisagem fluvial, sendo a paisagem fluvial compreendida como o habitat formado pelo curso d'água e os processos a ele associados. Considerou-se que esta integridade abrange aspectos hidrológicos, geomorfológicos e ecológicos representados em diferentes níveis de detalhamento com emprego de indicadores apropriados. Alterações da estrutura física, conservação da vegetação ripária, regime de escoamento e qualidade da água são exemplos de parâmetros encontrados nestes protocolos. O desempenho de cada procedimento foi analisado por intermédio da comparação dos resultados dos protocolos entre si e com os resultados de qualidade da água e da diversidade da fauna bentônica.

Essa abordagem preenche uma lacuna na caracterização da geomorfologia das paisagens, que se refere à escala de trecho de rio, porém a avaliação e monitoramento das características físicas do habitat fluvial nessa escala carecem de padronização (Fernández et al., 2011). Assim, apesar da adaptação ser necessária em muitas circunstâncias, também há a necessidade de adotar certo grau de uniformidade nas estratégias de avaliação, para facilitar posteriormente o monitoramento. Destaca-se que o trabalho não visa a estabelecer qual é o melhor protocolo, mas mostrar e discutir a aplicação, os aspectos mais bem abordados e os pontos que podem ser melhorados. O trabalho pode contribuir na escolha de técnicas para novos estudos, além de colaborar com a discussão e aprimoramento deste tipo de abordagem.

\section{MATERIAL E MÉTODOS}

\section{1. Área de estudo e desenho amostral}

A bacia hidrográfica do Itacorubi se localiza em Florianópolis na porção central da ilha, entre as coordenadas $\mathrm{x}=743.137 \mathrm{~m} ; \mathrm{y}=6.941 .075 \mathrm{~m} \mathrm{e} \mathrm{x}=749.568 \mathrm{~m} ; \mathrm{y}=6.948 .682 \mathrm{~m}$ (Fuso 22S, Datum SAD-69). Ocupa a área de $27,7 \mathrm{~km}^{2}$, considerando como parte da bacia toda a drenagem que desemboca no manguezal do Itacorubi (Figura 1).

Os cursos d'água Córrego Grande, rio do Meio, Ana d'Ávila e Itacorubi são os principais formadores da drenagem natural desta bacia. Nestes rios foram escolhidos os trechos de amostragem, utilizando-se os critérios de semelhança na ordem de rio $\left(3^{\mathrm{a}}\right.$ e $4^{\mathrm{a}}$ ordem) e na área da bacia $(1,0$ a 3,5 km²). Estes critérios são relevantes para possibilitar a comparação dos dados e a aplicabilidade dos métodos. Além disso, considerou-se a localização dos trechos relativamente à ocupação urbana em áreas próximas, o que permitiu variabilidade de condições ambientais a serem avaliadas. O estudo procurou representar dentro do possível as condições da bacia hidrográfica em questão, considerando as limitações financeiras, de esforço amostral e de processamento dos dados, principalmente de fauna bentônica.

Na Figura 1 são identificados os seis trechos de amostragem: um no rio Itacorubi (IT); um no rio Ana d'Ávila (AD); dois no rio do Meio (M1) e (M2); e dois no rio Córrego Grande (C1) e (C2), todos com comprimento de $100 \mathrm{~m}$. Os trechos se localizam no que se pode denominar de curso superior e médio dos rios, caracterizados por altas declividades e velocidades. Não foi realizada aplicação no ambiente estuarino, para isso seria necessária adaptação ou escolha de procedimentos específicos para essas áreas. No início dos trechos foram medidos parâmetros de qualidade da água e coletadas amostras da fauna bentônica, para efetuar a comparação com os resultados finais dos protocolos.

\subsection{Protocolos de Avaliação Rápida}

Os procedimentos escolhidos para caracterizar a integridade da paisagem fluvial foram: o IHF - índice do Habitat Fluvial; o PAH - Protocolo Simplificado de Avaliação de Habitats; e o procedimento de avaliação da condição dos cursos d'água urbanos, que ao longo do seu 
processo de aperfeiçoamento foi denominado Índice de Integridade (InI). Os parâmetros avaliados por cada protocolo são apresentados na Tabela 1, informações específicas sobre a forma de aplicação podem ser obtidas diretamente nas referências.
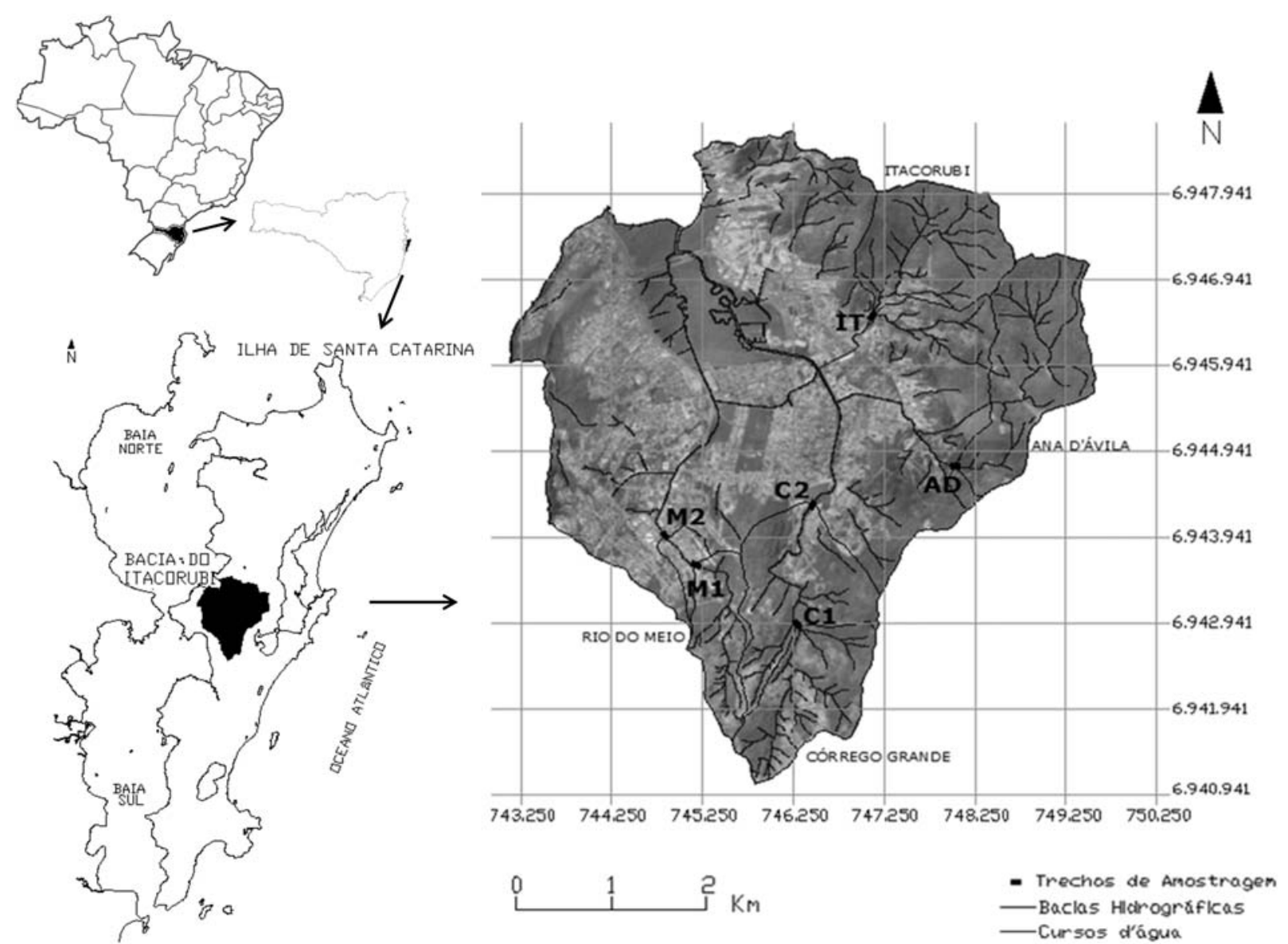

Figura 1. Localização da área de estudo - bacia hidrográfica do Itacorubi em Florianópolis-SC-Brasil - e dos trechos de amostragem em quatro cursos d'água pertencentes à bacia: nos rios Córrego Grande (C1 e C2), do Meio (M1 e M2), Ana d'Ávila (AD) e Itacorubi (IT).

O IHF caracteriza e pontua aspectos físicos do leito relacionados com a heterogeneidade e complexidade de habitats e que dependem em grande medida da hidrologia e do substrato existente (Pardo et al., 2002). Este procedimento tem sido frequentemente utilizado na Espanha e, para sua aplicação neste trabalho, realizou-se a tradução e adaptação dos termos utilizados, principalmente os relativos à vegetação aquática, tarefa que contou com o apoio de especialistas na área.

O PAH é uma modificação do protocolo elaborado por Hannaford et al. (1997), realizada por Callisto et al. (2001), para que ele seja utilizado em ecossistemas lóticos com diferentes níveis de preservação das características naturais, ou sob a influência antropogênica. Seu uso fornece uma avaliação geral e qualitativa de vários atributos dos habitats (Callisto et al., 2001).

O InI é resultado de pesquisas sobre a revitalização de cursos d'água em área urbana (Dalla Costa e Pompêo, 2008; Pompêo et al., 2011) com objetivo de quantificar e qualificar a integridade dos rios. O procedimento completo envolve a descrição qualitativa de cada trecho e a aplicação em todo o percurso do rio, visando obter comparações para indicação de ações prioritárias de revitalização, porém no escopo deste trabalho o protocolo foi aplicado apenas ao trecho de interesse. 
A fim de tornar possível a análise comparativa entre os protocolos, realizou-se uma parametrização da escala de seus resultados finais de forma que fossem representadas com valores entre 0 e 100 . Portanto, não se efetuou a comparação específica entre cada parâmetro que compõe os procedimentos e, sim, o resultado final de cada um dos protocolos.

Todos os protocolos foram aplicados nos dias 10 e 11 de novembro de 2014, uma vez que a avaliação das modificações sazonais não fez parte do estudo. A análise da variabilidade entre os resultados, caso os procedimentos fossem utilizados por diferentes pessoas com ou sem treinamento prévio não foram abordadas neste trabalho. Essa análise já foi discutida, entre outros, por Callisto et al. (2002) e Guimarães et al. (2012), os quais mostraram que não houve diferença significativa entre as aplicações.

\subsection{Qualidade da água}

Em cada local de amostragem foram medidos parâmetros in situ: temperatura e condutividade (Condutivímetro YSI-EC 300); oxigênio dissolvido (Oxímetro YSI - 55); pH (pHmetro YSI- 100 AC). Em seguida, coletaram-se amostras de água que foram acondicionadas, mantidas no escuro e sob refrigeração até os procedimentos em laboratório. As amostras de água foram homogeneizadas e, com uma alíquota, medida a turbidez (Turbidímetro Alfa-Kit ${ }^{\circledR}$ ). O restante de cada amostra foi filtrado e congelado para a análise de fósforo inorgânico dissolvido e pigmentos fitoplanctônicos, seguindo a metodologia de Grasshoff et al. (1999). Os pigmentos (clorofila-a e feoftina-a) foram extraídos com etanol $80 \%$. Os parâmetros foram escolhidos por serem efetivos na identificação de alterações da qualidade da água geradas pela urbanização, principalmente a eutrofização, e por permitirem o cálculo do Índice de Estado Trófico (IET).

\subsection{Macroinvertebrados bentônicos}

Os macroinvertebrados foram coletados com rede do tipo Surber, malha de $500 \mathrm{~m}$ de abertura. As amostras, fixadas em campo e conservadas com álcool $70 \%$, foram triadas no laboratório em bandeja e sob microscópio estereoscópico (total de 6 amostras por local). Os organismos foram contados e identificados até o menor nível taxonômico possível. Os principais guias de identificação seguidos foram: Mugnai et al. (2010); Merritt e Cummins (1984). Adicionalmente, após o processo de triagem da fauna, efetuou-se a análise de biomassa e teor de matéria orgânica com o substrato que ficou retido na rede de coleta.

\subsection{Análise dos dados}

A comparação dos resultados foi efetuada através da relação entre os protocolos e a avaliação das condições básicas da cadeia alimentar aquática (estado trófico) e da qualidade do habitat para os demais níveis, com ênfase na macrofauna bentônica. Para o tratamento dos dados foram utilizadas ferramentas da estatística multivariada, que permitem a visualização integrada dos resultados tanto dos índices como dos parâmetros de qualidade da água.

Para avaliar a condição de eutrofização dos cursos d'água, estimou-se o Índice de Estado Trófico (IET), adaptado por Lamparelli (2004). Os valores do IET são distribuídos em seis classes: ultraoligotrófico (IET $\leq 47)$; oligotrófico $(47<$ IET $\leq 52)$; mesotrófico $(52<$ IET $\leq$ 59); eutrófico (59 < IET $\leq 63)$; supereutrófico $(63<$ IET $\leq 67)$; hipereutrófico (IET > 67). Este índice fornece informações sobre a comunidade fitoplanctônica associada à concentração de nutrientes (Lamparelli, 2004). Assim, em conjunto com os outros parâmetros medidos, o IET indica as alterações na cadeia trófica, resultantes da urbanização da bacia hidrográfica a montante dos trechos de rio estudados. Além disso, constitui-se de uma alternativa proposta por Fasola et al. (2011) para quantificar o parâmetro que avalia a poluição no curso d'água, no protocolo de avaliação da integridade (InI). 
Tabela 1. Parâmetros que compõe cada protocolo: Índice do Habitat Fluvial (IHF), de acordo com Pardo et al. (2002); Protocolo Simplificado de Avaliação do Habitat (PAH), conforme Callisto et al. (2001); Índice de Integridade (InI), segundo Pompêo et al. (2011).

Índice do Habitat Fluvial (IHF)

Inclusão de corredeiras - sedimentação em poços

Frequência de corredeiras

Composição do substrato

Regimes de velocidade/profundidade

Porcentagem de sombra no leito

Elementos de heterogeneidade

Cobertura de vegetação aquática

Protocolo Simplificado de Avaliação do Habitat (PAH)
Tipos de fundos (peixes)
Frequência de remansos (ou curvas)
Tipos de substrato
Deposição de lama
Depósitos sedimentares
Alteração no canal do rio
Características do fluxo das águas
Presença de vegetação ripária
Estabilidade das margens
Extensão da vegetação ripária

Índice de Integridade (InI)

Corte ou remoção da vegetação

Substituição parcial ou total da vegetação nativa por espécies exóticas

Processos erosivos visíveis nas margens

Presença de sedimentos nas margens

Obstruções do leito por acúmulo de sedimentos

Retificação da seção transversal

Mudança de revestimento da seção transversal

Canalização ou tamponamento do trecho

Corte de meandros

Retirada da água sem modificação estrutural do curso d'água

Retirada da água com modificação estrutural do curso d'água

Obstruções por pontes e travessias inadequadas

Presença de resíduos sólidos depositados nas margens ou no leito menor do curso d'água

Lançamento de efluentes, conexões de esgoto

Lançamento de águas pluviais urbanas

Presença de edificações residenciais, comerciais, industriais ou da administração pública

Presença de áreas de esporte, lazer ou infraestrutura pública

A expressão da diversidade de organismos que indica a qualidade do habitat é complexa, portanto para a avaliação da comunidade de macroinvertebrados foram calculados vários índices: número de espécies $(\mathrm{S})$; número de indivíduos $(\mathrm{N})$; índice de riqueza de espécies de Margalef (d); índice de Pielou (J'); índice de Shannon-Wiener (H'); e índice de Simpson

Rev. Ambient. Água vol. 11 n. 1 Taubaté - Jan. / Mar. 2016 
(1- $\lambda$ ), no programa PRIMER 6. Adicionalmente, calculou-se o índice EPT composto da porcentagem dos taxa Ephemeroptera, Plecoptera e Trichoptera, considerados grupos sensíveis à poluição; e, em contraposição, a porcentagem do grupo oligochaeta, que é considerado um grupo resistente de organismos (Ferreira et al., 2012).

A relação entre todos os resultados foi avaliada pelo escalonamento multidimensional não-métrico (n-MDS), um método de ordenação que permite a visualização das relações de similaridade entre os locais. Desta forma, os resultados do estudo (parâmetros de qualidade da água, IET e abundância da fauna) são utilizados como dados de entrada para gerar a análise. Os resultados dos protocolos não foram utilizados para gerar os diagramas por uma questão de balanceamento de dados, pois para os parâmetros e dados de abundância há um número maior de amostras do que o número de aplicação dos protocolos.

Dois diagramas foram gerados, nos quais se sobrepôs o coeficiente de correlação de Spearman $(\rho)$. O tamanho dos vetores representa o valor do coeficiente, e o raio do círculo é o valor máximo $(\rho=1)$, foram representados os dados com $\rho>0,70$. O primeiro nMDS foi efetuado com os resultados dos parâmetros qualidade da água e do IET. Esses dados foram submetidos à normalização e com eles foi gerada a matriz de dissimilaridade (a partir da distância Euclidiana). Neste primeiro diagrama, o coeficiente de Spearman relaciona os resultados dos protocolos aos dados que foram utilizados para gerar o nMDS, ou seja, ele mostra a correlação entre os resultados dos protocolos com os parâmetros qualidade da água e o IET. O segundo nMDS foi realizado utilizando-se os dados de abundância da fauna, para os quais se aplicou a transformação logarítmica, a matriz gerada foi baseada na distância BrayCurtis. Neste diagrama o coeficiente de Spearman correlaciona os resultados dos protocolos e dos índices de diversidade aos dados de abundância da fauna. Deste modo, aqui a correlação foi calculada entre uma matriz de resultados (índices bióticos e protocolos), que é diferente dos dados utilizados para gerar o nMDS (dados de abundância).

Por fim, os resultados finais padronizados para todos os protocolos foram representados graficamente. Também foram apresentados os resultados numéricos dos coeficientes de correlação entre cada protocolo e entre os protocolos e os índices, para os índices com valores mais representativos. Os gráficos e as análises de correlação foram feitas no programa $\mathrm{R}$ (R Core Team, 2015).

\section{RESULTADOS E DISCUSSÃO}

Os protocolos se mostraram representativos na caracterização da integridade da paisagem fluvial nos trechos de amostragem, identificando diferenças entre esses locais conforme sua integridade. De acordo com os parâmetros de qualidade da água e o IET, o primeiro nMDS apresentou uma distribuição indicando um gradiente dos trechos menos poluídos para os mais poluídos, (Figura 2-a). Pela sobreposição do coeficiente, percebe-se que houve correlação positiva entre os resultados dos protocolos e dos parâmetros: oxigênio dissolvido e matéria orgânica particulada; e correlação inversa com o Índice de Estado Trófico - IET. Já no segundo nMDS, para os dados de fauna, houve uma diferenciação maior em dois grupos (Figura 2-b), um formado pelos trechos de rio com maior influência da área urbana (IT, M2 e C2) e outro dos trechos sob menor influência (AD, M1, C1). Neste nMDS o índice que melhor se correlacionou diretamente foi o índice Ephemeroptera, Plecoptera e Trichoptera EPT e inversamente o número de indivíduos $(\mathrm{N})$.

Apesar do segundo nMDS, relativo aos dados de abundância, ter mostrado o agrupamento entre os trechos mais urbanizados, mesmo entre eles observou-se diferenças nos valores dos índices de diversidade da fauna (Tabela 2). Isso mostra a importância de se utilizar várias métricas na estimativa desta diversidade. $\mathrm{O}$ trecho $\mathrm{C} 1$ no rio Córrego Grande se 
destaca nos valores dos índices EPT e riqueza de espécies (d). Já para a porcentagem do grupo oligochaeta, o rio Itacorubi (IT) se sobressai, uma vez que este valor represente, na média das amostras, $46 \%$ dos organismos encontrados.
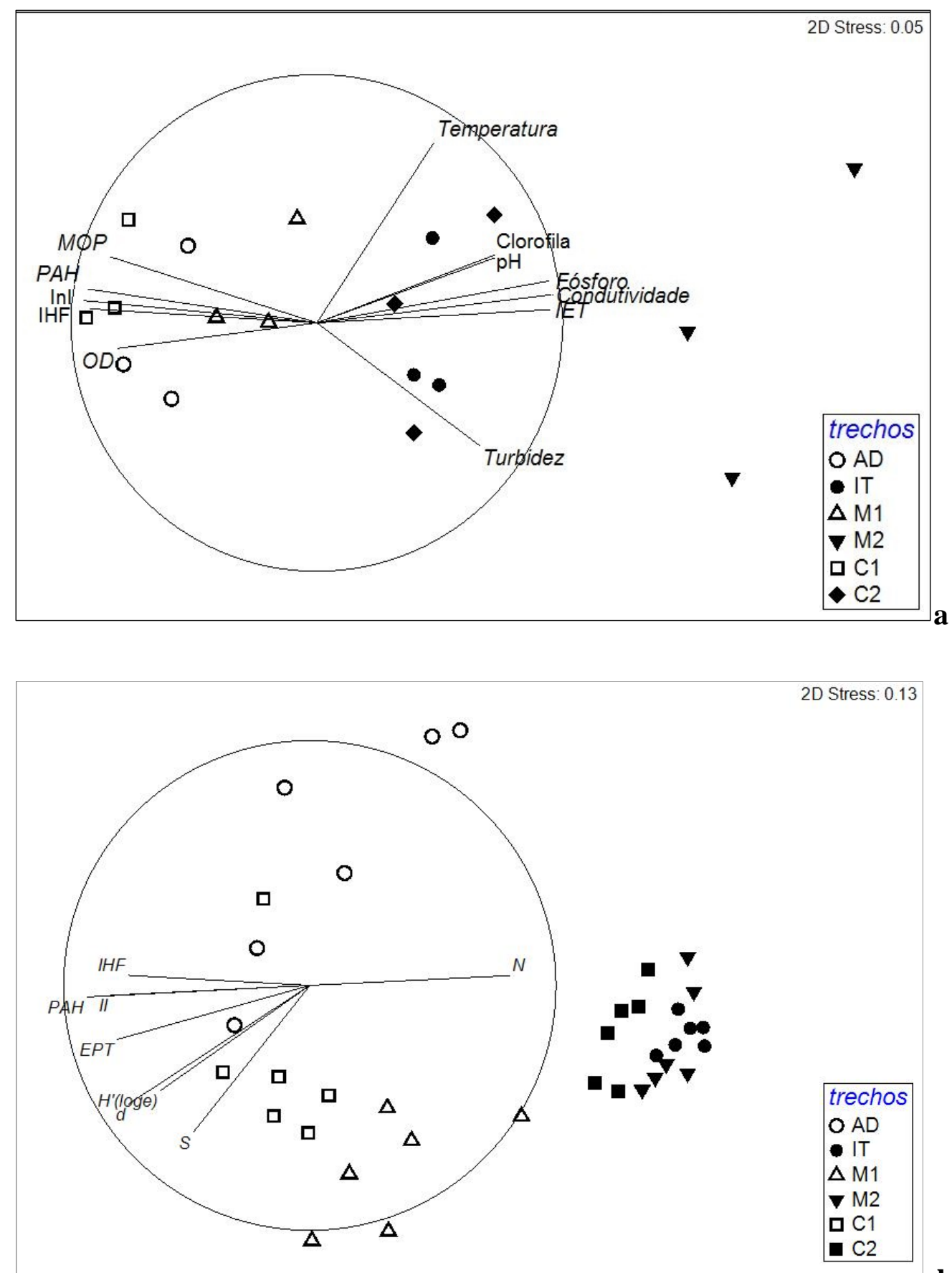

Figura 2. a) nMDS para os resultados de qualidade da água $(\mathrm{n}=3)$, sendo IHF (índice do Habitat Fluvial), PAH (Protocolo Simplificado de Avaliação de Habitats) e InI (Índice de Integridade) os protocolos e os parâmetros abreviados são: OD: oxigênio dissolvido; MOP: matéria orgânica particulada. Os parâmetros $\mathrm{pH}$ e clorofila ficaram sobrepostos na análise. b) nMDS para os dados de abundância $(n=3)$, sendo IHF, PAH e InI os protocolos e as métricas são: S: número total de espécies; N: número total de indivíduos; d: índice de riqueza de espécies (Margalef); J': índice de homogeneidade de Pielou; H': índice de diversidade de Shannon-Wiener; 1- $\lambda$ ': índice de Simpson. 
Tabela 2. Resultados gerais dos índices calculados para os trechos de amostragem, sendo os trechos: AD- no rio Ana d'Ávila; IT- no rio Itacorubi; C1 e C2- no rio Córrego Grande; M1 e M2- no rio do Meio. Índice de Estado Trófico (IET) para a qualidade da água e para a fauna: número total de espécies $(\mathrm{S})$; número total de indivíduos $(\mathrm{N})$; índice de riqueza de espécies (Margalef) (d); índice de homogeneidade de Pielou (J'); índice de diversidade de Shannon-Wiener (H'); índice de Simpson (1- $\lambda$ ); Porcentagem de Ephemeroptera, Plecoptera e Trichoptera (EPT); Porcentagem de Oligochaeta (oligo).

\begin{tabular}{cccccccccc}
\hline Trechos & IET & $\mathrm{S}$ & $\mathrm{N}$ & $\mathrm{d}$ & $\mathrm{J}$ & $\mathrm{H}^{\prime}$ & $1-\lambda$ & $\mathrm{EPT}$ & oligo. \\
\hline $\mathrm{C} 1$ & 46 & 14 & 102 & 2,87 & 0,74 & 1,95 & 0,78 & 27,8 & 1 \\
$\mathrm{AD}$ & 47 & 7 & 247 & 1,36 & 0,53 & 1,03 & 0,52 & 4,5 & 0 \\
$\mathrm{M} 1$ & 50 & 13 & 200 & 2,42 & 0,64 & 1,62 & 0,69 & 9,1 & 4 \\
$\mathrm{IT}$ & 56 & 9 & 2148 & 1,00 & 0,48 & 1,01 & 0,55 & 0,5 & 46 \\
$\mathrm{C} 2$ & 59 & 10 & 1360 & 1,30 & 0,37 & 0,83 & 0,39 & 0,2 & 11 \\
M2 & 67 & 6 & 1322 & 0,66 & 0,49 & 0,82 & 0,45 & 0,0 & 14 \\
\hline
\end{tabular}

A correlação mostrou valores acima de 0,80 para os três protocolos em relação ao Índice de Estado Trófico (IHF x IET $-\rho=0,92$; PAH x IET $-\rho=0,90$; InI x IET $-\rho=0,95$ ) e ao índice Ephemeroptera, Plecoptera e Trichoptera (IHF x EPT $-\rho=0,82$; PAH x EPT $-\rho=0,81$; InI x EPT $-\rho=0,86$ ). Esses resultados apontam para a efetividade dos protocolos na avaliação de trechos de rios distintos em termos de grau trófico e composição da fauna.

Os valores padronizados de cada protocolo, para os locais correspondentes, mostraram que houve uma mesma tendência de resultados (Figura 3). Isso se comprova pelo valor da correlação dos protocolos entre si: IHF x PAH: $\rho=0,899$; IHF x InI: $\rho=0,986$; PAH x InI: $\rho=0,943$. Contudo, percebe-se pelo gráfico que houve variação entre os resultados dos protocolos para um mesmo trecho, principalmente no trecho M2 do rio do Meio.

Colocando-se os locais com os valores dos índices em ordem decrescente, da maior para a menor integridade, verificou-se que todos os protocolos reconheceram os mesmos trechos de maior integridade: C1 (no rio Córrego Grande) e AD (no rio Ana d'Ávila), localizados a montante das áreas mais urbanizadas. Também o trecho de menor integridade foi o M2 no rio do Meio, o qual se encontra canalizado com revestimento em concreto no fundo e pedra argamassada nas margens. Já os trechos C2 e IT, no rio Córrego Grande e no rio Itacorubi, respectivamente, apresentaram muita similaridade entre si. Apesar de não ter sido feito teste estatístico, a similaridade foi observada pelo nMDS. Nesta análise não houve diferenciação entre as amostras desses trechos e, do mesmo modo, no IET os trechos C2 e IT apresentaram grau mesotrófico. Quanto à posição dos trechos na ordenação, é compreensível que não houvesse unanimidade entre os protocolos, pois eles mostraram condições de integridade muito semelhantes.

Embora se tenha percebido a consistência entre os resultados das análises e coeficientes de correlação quanto aos resultados dos protocolos, é relevante discutir algumas particularidades que refletem a aplicabilidade desses protocolos na avaliação da qualidade do habitat em trechos de rios urbanos. No IHF, que prioriza as características físicas do habitat, os trechos: M1 no rio do Meio e IT no rio Itacorubi obtiveram a mesma pontuação. Esse resultado pode ser atribuído ao fato de que a diferença entre esses locais está na qualidade da água e não propriamente na estrutura física. Esta diferença foi indicada no IET, em que o trecho M1 se caracterizou como oligotrófico e o trecho IT como mesotrófico. 


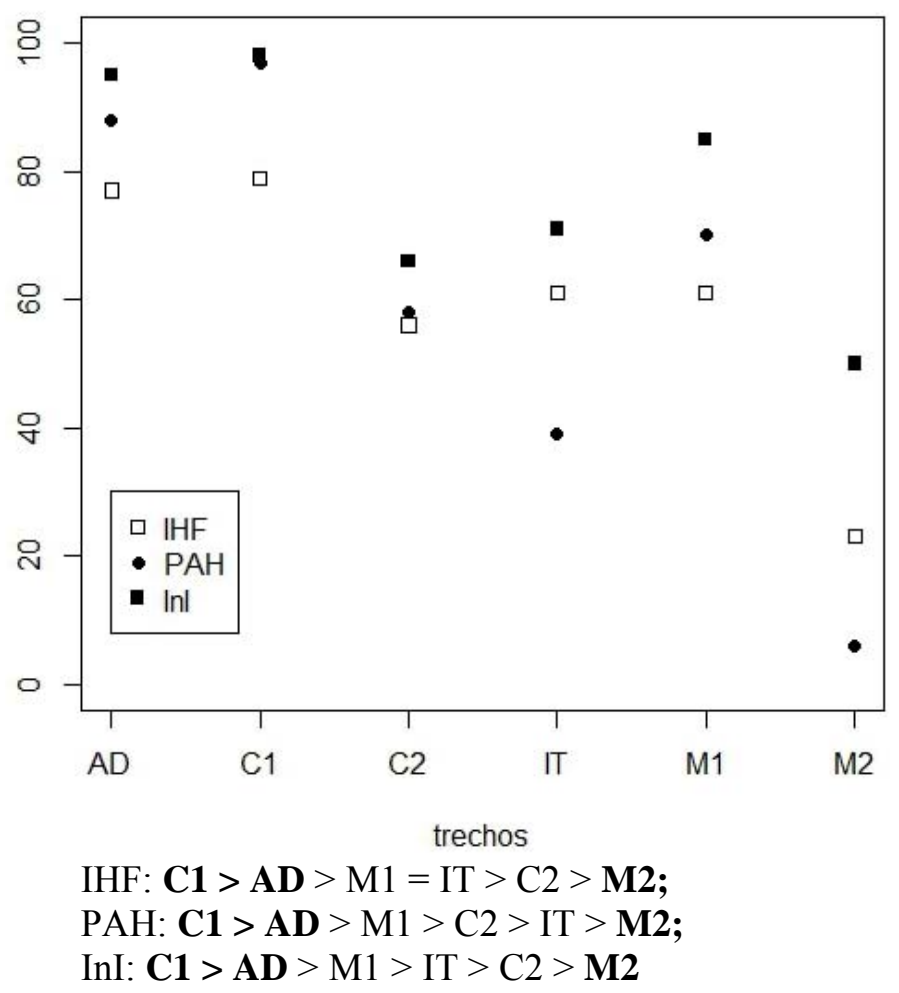

Figura 3. Gráfico dos valores finais dos protocolos, sendo os trechos: AD- no rio Ana d'Ávila; IT- no rio Itacorubi; $\mathrm{C} 1$ e $\mathrm{C} 2-$ no rio Córrego Grande; $\mathrm{M} 1$ e M2- no rio do Meio. Valores dos índices em ordem decrescente, da maior para a menor integridade.

Nota-se que o InI possui valores mais otimistas em relação aos outros dois protocolos. Isso se deve, em parte, pela sua formulação que foi baseada nos impactos sofridos nos cursos d'água, no contexto de degradação gerada pela urbanização. No meio urbano, um curso d'água considerado íntegro não necessariamente é isento de toda e qualquer alteração, como seria um ambiente de referência em conservação. Para o IHF e o PAH, por seu turno, a referência é um local sem perturbações; sua aplicação em área urbana tende então a gerar valores menores, pois a urbanização geralmente é acompanhada de fortes modificações na estrutura dos rios.

Em relação à fauna, os valores das correlações com os protocolos são menores, apontando para o fato de que os organismos não possuem respostas diretas como os parâmetros de qualidade da água, pois há inúmeros fatores interferentes e grande complexidade em quantificá-los. Por tal motivo, a avaliação da variedade de atributos do habitat, juntamente com outros índices, é crítica para a interpretação dos dados bióticos (Barbour et al., 1999). Além disso, é de fundamental importância a análise conjunta com o uso de várias métricas, bem como a relevância da qualificação dos grupos de organismos. Apenas a riqueza de taxa presentes e as características puramente físicas do habitat não fornecem tudo sobre a diversidade do local, pois há fatores relacionados ao ciclo de vida, às preferências e às tolerâncias biológicas dos organismos (Diaz et al., 2004).

A perspectiva de habitat proposta por Diaz et al. (2004), na qual o habitat é mais do que o substrato ou estrutura, é fundamental para a compreensão dos resultados. Embora a estrutura física seja elemento-chave, há as outras qualidades de apoio, como o suporte trófico e a biologia dos organismos em si. Desta forma, os protocolos que contemplam aspectos da 
qualidade da água são mais completos. No caso, o protocolo InI contempla esse aspecto no parâmetro lançamento de efluentes, inclusive com possibilidade de quantificação (Fasola et al., 2011). Contudo, este protocolo não aborda algumas características físicas essenciais para o habitat da fauna, como o regime de velocidade, a composição do substrato e a heterogeneidade, quesitos estes que são melhor abordados no IHF e PAH.

A maneira que os autores Callisto et al. (2002) encontraram de suprir a necessidade da parte de qualidade da água foi a aplicação conjunta do $\mathrm{PAH}$ com outro protocolo rápido, desenvolvido pela EPA (US Environmental Protection Agency). A adaptação e a aplicação de protocolos associados para complementar a avaliação, nesse sentido, revelam-se bastante viáveis. Pode-se dizer o mesmo da melhoria de protocolos existentes. Eles têm potencial para aumentar o nível de detalhamento, com inclusão de outros aspectos, sem que necessariamente passem a envolver os mesmos custos dos procedimentos analíticos convencionais. Afinal, os protocolos possuem também o objetivo de aproximar a linguagem científica, muitas vezes complicada, das necessidades enfrentadas pelos gestores e das expectativas das pessoas (Gregory et al., 2008; Guimarães et al., 2012), tornando mais fácil a apropriação social do saber científico.

O uso dos protocolos oferece uma visão geral da qualidade ambiental, que pode ser utilizada pelos gestores de recursos hídricos como instrumentos complementares (Rodrigues e Castro, 2008). Além disso, também são empregados: como instrumentos de educação ambiental (Guimarães et al., 2012); na formação profissional (Callisto et al., 2002); na caracterização hidromorfológica na escala de trecho de rio (Fernández et al., 2011); no monitoramento ambiental (Pardo et al., 2002) e na perspectiva de recuperação de rios (Habberfield et al., 2014). Isso ilustra a gama de aplicação e a utilidade da ferramenta. Os protocolos estão em constante aperfeiçoamento; testar sua aplicabilidade faz parte desse processo de melhoria.

\section{CONCLUSÃO}

Os três protocolos aplicados - Índice do Habitat Fluvial (IHF), Protocolo Simplificado de Avaliação de Habitat (PAH) e Índice de Integridade (InI) - mostraram bom desempenho para o reconhecimento da integridade do habitat fluvial. A escolha do método mais adequado depende dos objetivos do estudo, pois cada um possui suas particularidades e limitações. $\mathrm{O}$ IHF caracteriza melhor a estrutura física do rio, porém a integridade do habitat também depende da qualidade da água, item que é melhor avaliado pelos outros protocolos (PAH e InI). O emprego de técnicas associadas ou em conjunto com procedimentos analíticos torna o estudo mais completo. No entanto, a similaridade dos resultados, dos protocolos entre si e com as demais análises, mostrou a viabilidade de sua utilização na área de estudo. Para dar continuidade a essa perspectiva de trabalho poderão ser realizadas outras aplicações, que permitam a validação por teste estatístico, incluindo um maior número de trechos. Utilizar estes e outros procedimentos se mostra necessário para testar e avaliar a validade dos métodos e, desta forma, contribuir com a melhoria e padronização das técnicas de avaliação na escala de trecho de rio.

\section{AGRADECIMENTOS}

Agradecemos ao INCT-TMCOcean (projeto de pesquisa CNPq n ${ }^{\circ} 573.601 / 2008-9$ ) pelo apoio financeiro para realização dos campos, à Coordenação de Aperfeiçoamento Pessoal de Nível Superior (CAPES) pela bolsa de estudos e ao Programa de Pós-Graduação em Geografia da Universidade Federal de Santa Catarina (UFSC). 


\section{REFERÊNCIAS}

BARBOUR, M. T.; GERRITSEN, J.; SNYDER, B. D.; STRIBLING, J. B. Rapid bioassessment protocols for use in streams and wadeable rivers: periphyton, benthic macroinvertebrates and fish. 2. ed. EPA 841-B-99-002. Washington, D.C.: USEPA, 1999.

CALLISTO, M.; MORETTI, M.; GOULART, M. Macroinvertebrados bentônicos como ferramenta para avaliar a saúde de riachos. Revista Brasileira de Recursos Hídricos, v. 6, n. 1, p. $71-82,2001$.

CALlistO, M.; FERREIRA, W. R.; MORENO, P.; GOULART, M.; PETRÚCIO, M. Aplicação de um protocolo de avaliação rápida da diversidade de habitats em atividades de ensino e pesquisa (MG-RJ). Acta Limnologica Brasiliensia, v. 14, n. 1, p. 91-98, 2002.

DALLA COSTA, S.; POMPÊO, C. A. Revitalização de cursos d'água em áreas urbanas: caracterização e avaliação da degradação ambiental na zona de proteção legal do Rio Córrego Grande. In: SIMPÓSIO DE RECURSOS HÍDRICOS SUL-SUDESTE, 2., 2008, Rio de Janeiro. Anais... Rio de Janeiro: ABRH, 2008.

DIAZ, R. J.; SOLAN M.; VALENTE, R. M. A review of approaches for classifying benthic habitats and evaluating habitat quality. Journal of Environmental Management, v. 73, p. 165-181, 2004. http://dx.doi.org/10.1016/j.jenvman.2004.06.004

FASOLA, G. B.; SIMONASSI, J. C.; POMPÊO, C. A. Análise de variáveis físico-químicas e biológicas da água para um Protocolo de Avaliação Rápida da integridade de cursos d'água em áreas urbanas In: DIAS, V. L. N.; PET GEOGRAFIA UDESC (Orgs.). Cadernos do Observatório Geográfico da Grande Florianópolis do PET Geografia UDESC.1. ed. Florianópolis: Insular, 2011. p. 80-104.

FERNÁNDEZ, D.; BARQUÍN, J.; RAVEN, P. J. A review of river habitat characterisation methods: indices vs. characterisation protocols. Limnetica, v. 30, n. 2, p. 217-234, 2011.

FERREIRA, W. R.; RODRIGUES, D. N.; ALVES, C. B. M.; CALlisto, M. Biomonitoramento de longo prazo da Bacia do Rio das Velhas através de um índice multimétrico bentônico. Revista Brasileira de Recursos Hídricos, v. 17, n. 3, p. 253-259, 2012.

HANNAFORD, M. J.; BARBOUR, M. T.; RESH, V. H. Training reduces observer variability in visual-based assessments of stream habitat. Journal of the North American $\begin{array}{llllllll}\text { Benthological Society, v. 16, n. 4, p. } & \text { 853-860, }\end{array}$ http://www.jstor.org/stable/1468176

GUIMARÃES, A.; RODRIGUES, A. S. L.; MALAFAIA, G. Adequação de um protocolo de avaliação rápida de rios para ser usado por estudantes do ensino fundamental. Revista Ambiente \& Água, v. 7, n. 3, p. 241-260, 2012. http://dx.doi.org/10.4136/ambiagua.996

GRASSHOFF, K.; EHRHARDT, M.; KREMLING, K. Methods of seawater analysis. 3. ed. Weinheim: Verlag Chemie, 1999. 634 p. 
GREGORY, K. J.; BENITO, G.; DOWNS, P. W. Applying fluvial geomorphology to river channel management: Background for progress towards a palaeohydrology protocol. Geomorphology, v. 98, p. 153-172, 2008.

http://dx.doi.org/10.1016/j.geomorph.2007.02.031

HABBERFIELD, M. W.; BLERSCH, S. S.; BENNETT, S. J.; ATKINSON, J. F. Rapid geomorphic and habitat stream assessment techniques inform restoration differently based on levels of stream disturbance. Journal of the American Water Resources Association, v. 50, n. 4, p. 1051-1062, 2014. http://dx.doi.org/10.1111/jawr.12156

LAMPARELli, M. C. Grau de trofia em corpos d'água no estado de São Paulo avaliação dos métodos de monitoramento. 2004. 119 f. Tese (Doutorado em Ciências na Área de Ecossistemas Terrestres e Aquáticos) - Instituto de Biociências, Universidade de São Paulo, São Paulo, 2004.

MERRITT, R. W.; CUMMINS, K. W. (Orgs.) An introduction to the aquatic insects of North America. 2. ed. Iowa: Kendall/Hunt, 1984. 722 p.

MUGNAI, R.; NESSIMIAN, J. L.; BAPTISTA, D. F. Manual de identificação de macroinvertebrados aquáticos do estado do Rio de Janeiro. 1. ed. Rio de Janeiro: Technical Books, 2010. 176p.

OLlero, A.; ASKOA, I.; GONZALO, L. E.; ACÍN, V.; BALlARÍN, D.; DÍAZ, E. et al.The IHG index for hydromorphological quality assessment of rivers: updated version. Limnetica, v. 30, n. 2, p. 255-262, 2011.

PARDO, I.; ÁlVAREZ, M.; CASAS, J.; MORENO, J. L.; VIVAS, S.; BONADA, N. et al. El hábitat de los ríos mediterráneos. Diseño de un índice de diversidad de hábitat. Limnetica, v. 21, n. 3/4, p. 115-133, 2002.

POMPÊO, C. A.; RIGOTTI, J. A.; FREITAS FILHO, M. D. Urban stream condition assessment. In: INTERNATIONAL CONFERENCE ON URBAN DRAINAGE, 12. 2011, Porto Alegre. Anais... Porto Alegre: ABRH, 2011. p. 1-7.

R CORE TEAM. R: a language and environment for statistical computing. Vienna: $R$ Foundation for Statistical Computing, 2015.

RODRIGUES, A. S. L.; CASTRO, P. T. A. Protocolos de avaliação rápida: instrumentos complementares no monitoramento dos recursos hídricos. Revista Brasileira de Recursos Hídricos, v. 13, n. 1, p. 161-170, 2008.

SARAIVA, M. G. A. N.. O rio como paisagem: gestão de corredores fluviais no quadro do ordenamento do território. Lisboa: Fundação Calouste Gulbenkian, 1999. 512p.

WARD, J. V. Riverine landscapes: biodiversity patterns, disturbance regimes, and aquatic conservation. Biological Conservation, v. 83, n. 3, p. 269-278, 1998. http://dx.doi.org/10.1016/S0006-3207(97)00083-9 